\title{
RETRACTED ARTICLE: Empirical likelihood inference for the calibration regression model with lifetime medical cost
}

\author{
Yichuan Zhao • Min Lu
}

Received: 30 July 2009 / Accepted: 15 June 2010 / Published online: 6 July 2010

(C) Springer-Verlag 2011

This article has been retracted at the request of Y. Zhao, after learning that plagiarized text without appropriate quotation was found by the university in the thesis of co-author M. Lu. Major parts of the article were derived from the thesis. 